\title{
172 Malignant tumours of the paranasal sinuses
}

A Ohngren's line extends from the lateral canthus to the symphysis menti.

B Lederman's classification uses horizontal lines drawn through the superior and inferior orbital margins.

C Pain is the earliest clinical feature.

D Epiphora indicates involvement of the frontonasal duct.

E Biopsy is usually unnecessary as the diagnosis can be made radiologically.

\section{Treatment of malignant tumours of the paranasal sinuses}

A Sublabial antrostomy for biopsy is contraindicated because of the risk of tumour implantation.

$B$ If the tumour penetrates the cribriform plate it is inoperable.

C Radical surgery followed by radiotherapy gives 5 year survival figures of $80 \%$ overall.

D Orbital involvement is a contraindication to radical maxillectomy.

E Cytotoxic drug treatment is the first line of management.

\section{Pituitary tumours}

A Most pituitary tumours are malignant.

B Acromegaly is usually due to an acidophil adenoma.

C Cushing's syndrome can be treated by hypophysectomy.

D Transphenoidal hypophysectomy is particularly suitable for suprasellar tumours.

E The optic chiasma is at greater risk from transfrontal craniotomy than a transphenoidal approach. 